\title{
A connexin 26 mutation causes a syndrome of sensorineural hearing loss and palmoplantar hyperkeratosis (MIM 148350)
}

\author{
Kirsten Heathcote, Petros Syrris, Nicholas D Carter, Michael A Patton
}

\begin{abstract}
We report a missense mutation in the connexin 26 gene (GJB2) in a family with an autosomal dominant syndrome of hearing loss and hyperkeratosis. The affected family members have high frequency, slowly progressive, bilateral, sensorineural hearing loss and palmoplantar hyperkeratosis. The mutation causes an amino acid substitution (G59A), which may disrupt a reverse turn in the first extracellular loop of connexin 26. Connexin 26 mutations have been reported in syndromes of deafness and palmoplantar keratoderma. These data provide additional evidence for the role of connexin 26 in syndromes of this type.

(F Med Genet 2000;37:50-51)
\end{abstract}

Keywords: sensorineural hearing loss; palmoplantar hyperkeratosis; connexin 26

We have previously reported a three generation family with an autosomal dominant syndrome of palmoplantar hyperkeratosis and sensorineural hearing loss, which has been regarded as a distinct clinical entity (MIM number 148350$).{ }^{1}$ The bilateral hearing loss is moderate to severe in the high frequencies and progresses slowly after onset in early childhood. The hyperkeratosis of the palms and soles does not develop until mid childhood and is exacerbated by trauma.

Mutations in either the mitochondrial serine tRNA gene $(\mathrm{A} 7445 \mathrm{G})^{23}$ or the connexin 26 gene $^{45}$ have been associated with syndromes that involve both sensorineural hearing loss and palmoplantar keratoderma. Although the pedigree we present shows both female and male transmission of the syndrome, which is consistent with autosomal dominant inheritance, we have analysed and excluded the mitochondrial A7445G mutation. Richard et $a l^{4}$ reported a missense mutation in the connexin 26 gene, R75W, which segregates with a syndrome of dominant, prelingual, profound hearing loss and palmoplantar keratoderma. However, they also observed this mutation in an unrelated control without palmoplantar keratoderma, suggesting it may not be causative, although in vitro experiments have shown the R75W isoform of connexin 26 to inhibit gap junction conductance. More recently, a D66H substitution in connexin 26 has been reported in three unrelated families with Vohwinkel's syndrome, a syndrome of moderate to severe sensorineural deafness and mutilating keratoderma of the hands. ${ }^{5}$ Therefore, connexin 26 is a good candidate for this syndrome of hearing loss and hyperkeratosis.

We screened the coding exon of connexin 26 for mutations by SSCP in available members of the family previously described ${ }^{1}$ and identified two mutations. Sequence analysis of the coding region of connexin 26 showed both $\mathrm{C}$ and $\mathrm{G}$ at nucleotide 176 in all four of the affected subjects in the pedigree, whereas each of the unaffected subjects sequenced was homozygous for $G$ at this position (fig 1 ). This nucleotide substitution corresponds to a heterozygous glycine to alanine mutation at codon 59 (G59A) in the affected subjects (fig 2 ). The second mutation was a $\mathrm{C}$ to G substitution at nucleotide 249 , which causes a phenylalanine to leucine substitution at codon 83 (F83L). Two people in the pedigree were heterozygous for this change, the grandfather (I.1) and his son (II.4). F83L does not,

A

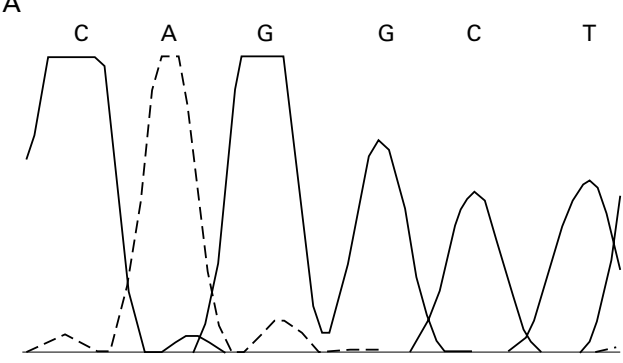

B

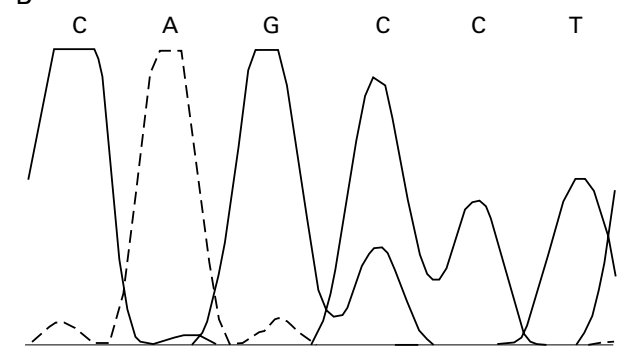

Figure 1 Sequence electrophoretograms showing nucleotides corresponding to 173-178 of the connexin 26 gene. The wild type sequence $(A)$ is from an unaffected family member (III.1) and the sequence showing the heterozygous $176 \mathrm{G}>C$ substitution (B) is from affected family member II.2. 


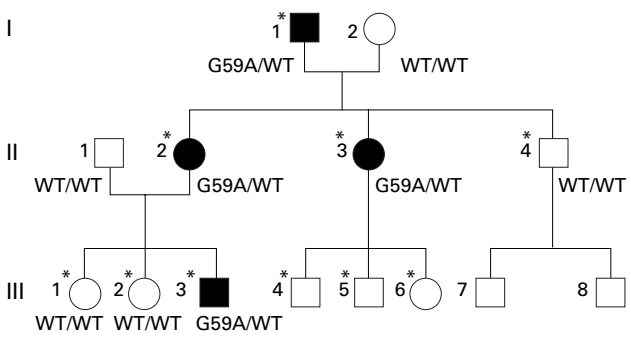

Figure 2 Pedigree shows the segregation of the G59A missense mutation with the syndrome. Subjects marked $\left(^{\star}\right)$ have undergone audiological examination. All of the affected subjects exhibit both moderate to severe hearing loss and palmoplantar hyperkeratosis. The extended pedigree is given in a previous publication, ${ }^{1}$ where III. 2 was incorrectly reported as being affected. Monitoring of the family over the last decade has shown her to be unaffected.

therefore, segregate with the syndrome. Additionally, F83L has been reported in subjects with normal hearing, suggesting that it is a neutral polymorphism. ${ }^{6}$ We have not observed G59A in 50 unrelated, unaffected, white controls and 55 unrelated white subjects with deafness but no palmoplantar hyperkeratosis nor has this missense substitution been published to date. We propose that G59A is causing the syndrome of hearing loss and hyperkeratosis observed in this family.

The G59A mutation is located in the first extracellular loop (E1) of connexin 26. The extracellular loop is highly conserved among the connexins and a study of connexin 32 proposed that the structure of the extracellular loop regions consists of stacked, antiparallel $\beta$ sheets, stabilised by disulphide bonds. ${ }^{7}$ The G59A mutation occurs in the motif QPGC, which is conserved among all $\alpha$ and $\beta$ connexins sequenced to date, and is approximately half way along the extracellular loop. It has been suggested that this motif is a reverse turn ${ }^{7}$; if this were so, then the substitution of a glycine residue with a bulkier alanine residue may disrupt the structure of the extracellular loop.
A gap junction is formed by the interaction of connexons on adjacent cells. Each connexon is a hexameric complex of connexin molecules and the first extracellular loop of the protein is thought to play a role in connexon-connexon interactions. This may explain why disruption of the structure of this domain could cause the autosomal dominant phenotype observed in this syndrome. It is interesting to note that the more severe phenotype of Vohwinkel's syndrome was caused by the $\mathrm{D} 66 \mathrm{H}$ mutation, ${ }^{5}$ which is also in this extracellular loop. These data, therefore, suggest that connexin 26 may be involved in many of the autosomal dominant syndromes of deafness and palmoplantar dermatological changes and the severity of the phenotype may be dependent upon the mutation involved.

This work was supported by a grant from the Birth Defects Foundation (UK)

1 Sharland M, Bleach NR, Goberdhan PD, Patton MA. Autosomal dominant palmoplantar hyperkeratosis and sensorineural deafness in three generations. $\mathcal{F}$ Med Genet 1992;29:50-2.

2 Reid FM, Vernham GA, Jacobs HT. A novel mitochondrial point mutation in a maternal pedigree with sensorineural deafness. Hum Mutat 1994;3:243-7.

3 Sevior KB, Hatamochi A, Stewart IA, et al. Mitochondrial A7445G mutation in two pedigrees with palmoplantar keratoderma and deafness. Am ₹ Med Genet 1998;75:17985.

4 Richard G, White TW, Smith LE, et al. Functional defects of $\mathrm{Cx} 26$ resulting from a heterozygous missense mutation in a family with dominant deaf-mutism and palmoplantar keratoderma. Hum Genet 1998;103:393-9.

5 Maestrini E, Korge BP, Ocana-Sierra J, et al. A missense mutation in connexin26, D66H, causes mutilating keratoderma with sensorineural deafness (Vohwinkel's syndrome) in three unrelated families. Hum Mol Genet 1999;8:123743.

6 Scott DA, Kraft ML, Carmi R, et al. Identification of mutations in the connexin 26 gene that cause autosomal recessive nonsyndromic hearing loss. Hum Mutat 1998;11: 387-94

7 Foote CI, Zhou L, Zhu X, Nicholson BJ. The pattern of disulphide linkages in the extracellular loop regions of connexin 32 suggests a model for the docking interface of gap junctions. F Cell Biol 1998;140:1187-97. 